\title{
Erros na classificação de touros, vacas e touros jovens geneticamente superiores avaliados na presença de heterogeneidade
}

\section{Antonio Policarpo Souza Carneiro1, Robledo de Almeida Torres², Paulo Sávio Lopes ${ }^{2}$, Ricardo Frederico Euclydes², Paulo Luiz Souza Carneiro ${ }^{3}$, Elizângela Emidio Cunha ${ }^{4}$}

\author{
1 Departamento de Informática - UFV, Viçosa, MG. \\ 2 Departamento de Zootecnia - UFV, Viçosa, MG. \\ 3 UESB, Jequié, $B A$. \\ ${ }^{4}$ Doutora em Genética e Melhoramento.
}

RESUMO - Foram simuladas quatro estruturas de dados com diferentes padrões de heterogeneidade entre rebanhos e com diferentes graus de conexidade genética. As estruturas simuladas diferiam quanto aos parâmetros heterogêneos entre rebanhos: 1) heterogeneidade para todos os parâmetros; 2) médias genéticas homogêneas e demais parâmetros heterogêneos; 3) heterogeneidade para parâmetros fenotípicos; e 4) rebanhos sem heterogeneidade. Após a predição dos valores genéticos, calculou-se a porcentagem de animais comuns entre dois grupos de classificação para os animais geneticamente superiores: grupo 1 - classificação com base nos valores genéticos verdadeiros e grupo 2 - classificação com base nos valores genéticos preditos. Para dados com heterogeneidade para todos os parâmetros e $0 \%$ de conexidade genética, a porcentagem de touros comuns aos dois grupos de classificação foi baixa e houve grandes erros na classificação dos touros geneticamente superiores. Quando os rebanhos possuíam 100\% de conexidade genética, esta porcentagem foi superior a $73 \%$ e os erros na classificação dos touros foram menores. Para vacas e touros jovens, mesmo para dados com 100\% de conexidade genética entre rebanhos, a predição dos valores genéticos foi muito afetada pela presença da heterogeneidade para todos os parâmetros. Para as estruturas sem heterogeneidade para média genética, as porcentagens de animais comuns entre os grupos de classificação foram altas e os erros na classificação dos animais geneticamente superiores foram pequenos, mesmo havendo heterogeneidade para outros parâmetros. Esses resultados sugerem que a heterogeneidade entre rebanhos para médias genéticas teve grande efeito sobre a acurácia da predição dos valores genéticos dos animais. Contudo, quando a heterogeneidade entre os rebanhos ocorreu para outros parâmetros como variância genética, média fenotípica e variância fenotípica, os resultados das avaliações genéticas dos animais foram próximos aos obtidos para dados sem heterogeneidade entre rebanhos.

Palavras-chave: avaliação genética, conexidade, seleção, simulação

\section{Errors in ranking of bulls, cows and young bulls evaluated in the presence of heterogeneity}

\begin{abstract}
Four structures of data with different degree of genetic connect ness and heterogeneity of parameters among herds: 1) heterogeneity of all parameters; 2) genetic means homogeneous and other parameters heterogeneous; 3 ) heterogeneity of phenotypic parameters and 4) no heterogeneity for herds, were simulated. After prediction of breeding values, the percentage of top animals common to group 1 (animals ranked by the true breeding values) and group 2 (animals ranked by the predicted breeding values) was calculated. For data with heterogeneity of all parameters and $0 \%$ of genetic connectness, the percentage of bulls common to the two groups was low and large errors were observed in ranking of top bulls. For $100 \%$ of genetic connect ness that percentage was larger than $73 \%$ and errors in ranking of top bulls were low. For cows and young bulls, even for data with $100 \%$ of genetic connect ness among herds, the prediction of breeding values was strongly affected by the heterogeneity of all parameters. For the structures with homogeneous genetic means, the percentages of common animals between groups were high and the errors in ranking of top animals were low, even under heterogeneity of the other parameters. These results suggest that heterogeneity of genetic means among herds strongly affects the accuracy of prediction of animals breeding values. However, results from genetic evaluations in the presence of heterogeneity among herds for genetic variance, phenotypic mean and variance were similar to those obtained for data without heterogeneity for these parameters.
\end{abstract}

Key Words: connectness, genetic evaluation, selection, simulation 


\section{Introdução}

As avaliações genéticas de bovinos freqüentemente precisam ser feitas em ambientes heterogêneos que podem diferir tanto em média quanto em variância. As diferenças entre rebanhos quanto à variabilidade de produção geralmente têm sido atribuídas às diferenças nos fatores climáticos locais ou regionais e nos tipos de manejo de cada rebanho.

Se as variâncias não são as mesmas entre os rebanhos com diferentes níveis tecnológicos, mas são assumidas como homogêneas, os animais podem ser classificados erroneamente, visto que as vacas de rebanhos superiores e os reprodutores com a maioria de suas filhas em rebanhos com maiores variâncias tenderiam a ter seus méritos genéticos superestimados. As produções das filhas provenientes de rebanhos mais variáveis teriam maior influência na avaliação do reprodutor, visto que a produção destas filhas é ponderada na proporção dos desvios-padrão dos rebanhos em que foram criadas (Torres et al., 2000).

A magnitude dos efeitos da heterogeneidade de variância depende da intensidade de seleção. Boldman \& Freeman (1990) encontraram maiores alterações na classificação de $1 \%$ das melhores vacas em relação aos $5 \%$ dos melhores reprodutores quando os dados foram ajustados para heterogeneidade de variância.

Vários estudos não têm apresentado grandes alterações na classificação dos animais em razão daheterogeneidade de variâncias. A correlação média de ordem entre os valores genéticos estimados em diferentes ambientes tem sido, geralmente, igual ou superior a 0,90 (Boldman \& Freeman, 1990; Canavesi et al., 1995). Meuwissen \& Van Der Werf (1993) verificaram que, após corrigir os dados para efeito de rebanho-ano, o ganho genético não reduziu em razão da heterogeneidade de variância.

Porém, outros trabalhos têm comprovado alteração na ordem de classificação dos animais quando considerada ou não a heterogeneidade de variância entre grupos genéticos nos modelos para predição dos valores genéticos. Segundo Crews \& Franke (1998), valores de correlações de ordem inferiores a 0,70 podem resultar em alterações nas classificações dos animais. Es tes autores encontraram correlações de ordem que variaram de 0,82 a 0,99 para classificações considerando ou não variâncias específicas para cada grupo genético. No trabalho de Oliveira et al. (2001), es ses valores de correlações de ordem variaram de 0,86 a 0,95 (os maiores foram obtidos para a característica peso aos 365 dias em relação a peso aos 550 dias). Os valores de correlação de ordem encontrados por Nuñez-Domingues et al. (1995) também indicaram alterações na classificação dos animais.
Depois de estimados os valores genéticos em análise de característica múltipla e considerando a seleção de 1 e $0,25 \%$ dos melhores touros, Weigel et al. (2001) encontraram baixa porcentagem de touros selecionados em comum entre alguns pares de países, como Alemanha e Estônia, Alemanha e Israel, Austrália e Itália, Suíça e Estônia, e Suíça e Itália. A porcentagem de touros selecionados em comum variou de 0,42 a 0,78 e de 0,39 a 0,78 para seleção de 1 e de $0,25 \%$ dos melhores touros, respectivamente. Os valores de porcentagem de touros selecionados em comum foram maiores entre países com sistema de produção semelhante, atingido valores superiores a 0,50. Entre países com sistema de produção diferentes, estas porcentagens variaram de 0,39 a 0,65 .

Segundo Weigel et al. (2001), algumas questões devem ser resolvidas para que a avaliação genética internacional de animais seja viável: primeiro, o número de touros comuns entre alguns países é muito reduzido, levando a estimativas de correlações pouco precisas, portanto, é preciso aumentar as ligações genéticas entre os vários países participantes destas avaliações; segundo, a inclusão de pequenos países ou de países com poucas informações contribui para elevar o custo computacional das avaliações, visto que cada país incluído representa uma nova característica e um conjunto de parâmetros a ser estimados. Uma alternativa citada pelos autores é o agrupamento dos países de acordo com o sistema de produção predominante.

Weigel \& Rekaya (2000) propuseram agrupar os rebanhos de diferentes países com base em variáveis descritivas que forneciam informações relativas a clima, manejo e composição genética de cada rebanho. Por meio da análise de agrupamento utilizando 13 variáveis descritivas, os autores agruparam os rebanhos de 13 diferentes países em cinco grupos, de modo que cada grupo correspondeu a uma variável. Nesta metodologia, os rebanhos são agrupados independentemente das fronteiras dos países, visto que rebanhos localizados em países pequenos e vizinhos são muito mais semelhantes que aqueles localizados em um mesmo país mas distantes um do outro e em regiões muito diferentes.

Os objetivos neste trabalho foram quantificar o efeito da heterogeneidade entre rebanhos para diferentes parâmetros sobre a classificação de touros, vacas e touros jovens geneticamente superiores e avaliar a relação entre heterogeneidade e conexidade genética.

\section{Material e Métodos}

Os dados utilizados neste trabalho foram simulados utilizando-se o sistema de simulação genética Genesys. O 
programa Genesys permite a simulação do genoma dos animais de determinada espécie. Para cada característica simulada, são definidos o número de locos e alelos envolvidos, o efeito dos genes, o número de efeitos fixos, a herdabilidade e os parâmetros genéticos e fenotípicos.

Neste trabalho, foi simulado um genoma de 2.000 centimorgans de comprimento para uma característica governada por 200 locos de dois alelos. As freqüências iniciais dos alelos foram simuladas com base na distribuição uniforme com média 0,50 . Os locos foram distribuídos em 15 pares de cromossomos autossômicos de tamanhos aleatórios. Os alelos possuíam apenas efeitos aditivos. Além do efeito de sexo, foi simulado o efeito de ambiente relativo a 15 rebanhos. Os efeitos de ambiente não controlados foram simulados conforme a distribuição normal.

Foram simulados os genomas de 4.500 fêmeas e de 4.500 machos que formaram a população-base. A partir da população-base, foram tomados aleatoriamente 75 machos e 3.750 fêmeas (um macho para 50 fêmeas), sendo obtidas dez progênies por fêmea, originando a população inicial com 37.500 progênies. Estes animais foram distribuídos em 15 rebanhos com parâmetros genéticos e fenotípicos heterogêneos ou não, de acordo com a estrutura de dados desejada.

Na população inicial, cada rebanho tinha 2.500 progênies, número que foi reduzido para 500 progênies/rebanho, totalizando 7.500 progênies no arquivo de dados disponível para análise. Esta redução no volume de dados foi ocasionada pela filtragem nos dados para gerar heterogeneidade para variâncias genéticas entre rebanhos. Após a classificação das 2.500 progênies de cada rebanho em ordem decrescente dos valores genéticos verdadeiros, tomaram-se 500 progênies mais afastadas umas das outras, obtendo-se rebanhos com maior variância genética, ou tomaram-se progênies mais próximas, obtendo-se rebanhos com menor variância genética. Assim, foram selecionadas 500 progênies espaçadas de 5 em 5 , de 3 em 3 e de 2 em 2 , para obtenção das estruturas de alta, média e baixa variância genética, respectivamente.

Com o objetivo de avaliar o efeito da heterogeneidade entre rebanhos sobre a classificação de animais geneticamente superiores, foram simuladas quatro estruturas de dados que diferiam quanto ao tipo de heterogeneidade genética e/ou fenotípica entre os rebanhos.

Na primeira estrutura de dados simulados, os rebanhos apresentavam heterogeneidade para todos os parâmetros considerados (RHTP). Foram simulados três diferentes níveis de rebanhos em termos de variância fenotípica: rebanhos de alta, média e baixa variância, cada nível com cinco rebanhos. As variâncias fenotípicas dos rebanhos, para cada nível de variabilidade, foram: alta (586 a 688), média (327 a 357) e baixa (195 a 213). Rebanhos com maiores variâncias fenotípicas também possuíam maiores médias genéticas e fenotípicas e maiores variâncias genéticas. As variâncias genéticas dos rebanhos para cada nível de variabilidade foram: alta (174 a 219), média (72 a 79) e baixa (39 a 46). As herdabilidades assumiram valores próximos entre os níveis de variabilidade, variando de 0,20 a 0,34 .

$\mathrm{Na}$ segunda estrutura de dados, os rebanhos apresentavam o mesmo padrão de heterogeneidade da primeira estrutura simulada. No entanto, as médias genéticas foram similares entre os níveis de variabilidade, ou seja, todos os rebanhos tinham animais com potencial genético semelhante (rebanhos com médias genéticas similares-RMGS). As variâncias fenotípicas dos rebanhos para cada nível de variabilidade foram: alta (876 a 991), média (521 a 652) e baixa (334 a 404), enquanto as variâncias genéticas foram: alta (436 a 529), média (282 a 382) e baixa (187 a 226). As herdabilidades assumiram valores próximos entre os níveis de variabilidade, variando de 0,49 a 0,58 .

Uma terceira estrutura de dados foi simulada semelhantemente à primeira, porém, neste caso, a heterogeneidade entre os rebanhos ocorreu apenas para os parâmetros fenotípicos (rebanhos com heterogeneidade fenotípica - RHF). Nesta estrutura, o aumento de variância fenotípica entre rebanhos foi acompanhado por aumento na média fenotípica, mas a média e a variância genética entre os diferentes níveis de variabilidade foram similares. As variâncias fenotípicas dos rebanhos, para cada nível de variabilidade, foram: alta (815 a 908), média (686 a 773) e baixa (597 a 731). Neste caso, como as variâncias genéticas foram similares entre os níveis de variabilidade, rebanhos com maior variância fenotípica apresentaram herdabilidades mais baixas. As herdabilidades variaram de 0,51 a 0,55 ; de 0,61 a 0,69 e de 0,72 a 0,81 para os rebanhos de alta, média e baixa variabilidade.

$\mathrm{Na}$ quarta estrutura de dados (rebanhos sem heterogeneidade - RSH), os rebanhos não apresentaram heterogeneidade para nenhum parâmetro. As médias fenotípicas e genéticas e as variâncias genéticas e fenotípicas dos rebanhos foram similares. As herdabilidades assumiram valores próximos entre os níveis de variabilidade, variando de 0,62 a 0,69.

Foram simulados dois conjuntos de dados em relação à conexidade genética ou à ligação genética entre os níveis de variabilidade para verificar a relação entre heterogeneidade e conexidade dos dados. A conexidade genética entre os níveis de variabilidade foi criada pelos touros com progênies em mais de um nível. 
No primeiro conjunto de dados, não houve conexidade genética entre os níveis de alta, média e baixa variabilidade (CG-0). Os touros utilizados em determinado nível de variabilidade não tinham progênies nos outros níveis.

Foi simulado um segundo conjunto de dados com 100\% de conexidade genética (CG-100) no qual todos os 75 touros tinham progênies nos três níveis de variabilidade.

A combinação das quatro estruturas de dados para heterogeneidade (RHTP, RMGS, RHF e RSH) e dos dois níveis de conexidade genética (CG-100 e CG-0) gerou oito arquivos de dados disponíveis para avaliação genética.

Os dados simulados nos oito arquivos, com diferentes graus de conexidade genética e de heterogeneidade entre rebanhos, foram utilizados para obtenção das estimativas de componentes de variância e herdabilidades utilizando-se o programa MTDFREML Estimação pelo Método da Máxima Verossimilhança Restrita adotando-se o procedimento Livre de Derivadas).

Os valores genéticos dos animais foram preditos utilizando-se o Modelo Animal considerando os efeitos fixos de sexo e rebanho, em análise de característica única considerando a presença de heterogeneidade genética ou fenotípica entre os rebanhos.

Para verificar o efeito da heterogeneidade sobre a classificação genética dos animais, foram selecionados 5 , 10 , e $20 \%$ dos touros e vacas geneticamente superiores e 1 e $5 \%$ dos melhores touros jovens, com base nos valores genéticos preditos. A categoria touros jovens refere-se a machos jovens que ainda não tiveram progênies. As mesmas proporções de seleção, citadas anteriormente, foram utilizadas para a seleção das diferentes categorias animais com base nos valores genéticos verdadeiros, conhecidos quando se trabalha com dados simulados pelo Genesys. Para as diferentes estruturas de dados, calculou-se a porcentagem de animais comuns aos dois grupos de classificação: grupo 1 - seleção com base nos valores genéticos verdadeiros; e grupo 2 - seleção com base nos valores genéticos preditos na avaliação genética.

A classificação dos animais geneticamente superiores e as porcentagens de animais comuns entre grupos de classificação foram obtidas pelo pacote estatístico Statistical Analisys System - SAS (Littel et al., 1995).

\section{Resultados e Discussão}

O tipo de heterogeneidade afetou as estimativas de variância. A variância genética estimada para a estrutura RHTP foi muito menor que a simulada, o que levou a estimativas de herbabilidades também muito baixas $(0,003$ a 0,10 ). Para as demais estruturas de dados, as estimativas de variâncias e herdabilidades assumiram valores intermediários aos simulados.

Considerando a estrutura de dados RHTP e CG-0, as porcentagens de touros comuns aos dois grupos de classificação foram baixas (Tabela 1). Para 5, 10 e 20\% dos melhores touros, as porcentagens de touros comuns aos dois grupos de classificação foram de 25,25 e $33 \%$, respectivamente. Estes resultados revelam que, na presença de heterogeneidade para todos os parâmetros e na ausência de conexidade genética dos dados, pequena porcentagem dos melhores touros seria corretamente identificada pela avaliação genética, o que compromete a eficiência da seleção e, conseqüentemente, o progresso genético. Esses erros na classificação dos melhores touros podem ocasionar grandes prejuízos nos programas de melhoramento, principalmente de gado de corte, nos quais touros que assumem as primeiras posições nas avaliações genéticas são utilizados intensivamente deixando grande número de descendentes.

Para a estrutura de dados RMGS, quando se consideraram 5, 10 e $20 \%$ dos melhores touros, as porcentagens de touros comuns aos dois grupos foram, respectivamente, de 50,50 e $73 \%$ para CG-0. O efeito deste tipo de heterogeneidade e da falta de conexidade entre os rebanhos foi grande sobre a porcentagem de animais comuns entreos

Tabela 1 - Porcentagens de animais em comum entre os dois grupos de classificação, com base no valor genético verdadeiro e no valor genético predito, para 5, 10 e $20 \%$ dos melhores touros

Table 1 - Percentage of common bulls in the two groups ranked by true or predicted breeding values for 5,10 and $20 \%$ of selection

Estrutura dos dados

Data structure

\begin{tabular}{lcccc} 
& $\begin{array}{c}\text { RHTP } \\
\text { HHAP }\end{array}$ & $\begin{array}{c}\text { RMGS }^{2} \\
\text { HSGM }\end{array}$ & $\begin{array}{c}\mathrm{RHF}^{3} \\
\text { HPH }\end{array}$ & $\begin{array}{c}\mathrm{RSH}^{4} \\
H W H\end{array}$ \\
\hline Seleção (\%) & \multicolumn{5}{c}{ CG-0 } \\
Selection(\%) & \multicolumn{4}{c}{ GC-0 } \\
\hline 5 & 25 & 50 & 75 & 75 \\
10 & 25 & 50 & 88 & 88 \\
20 & 33 & 73 & 93 & 73 \\
\hline Seleção (\%) & \multicolumn{5}{c}{ CG-100 } \\
Selection(\%) & \multicolumn{5}{c}{ GC-100 } \\
\hline 5 & 100 & 100 & 75 & 100 \\
10 & 88 & 88 & 88 & 88 \\
20 & 80 & 73 & 93 & 87 \\
\hline
\end{tabular}

${ }^{1}$ Rebanhos com heterogeneidade em todos os parâmetros

2 Rebanhos com médias genéticas similares.

3 Rebanhos com heterogeneidade fenotípica.

${ }^{4}$ Rebanhos sem heterogeneidade.

5 Conexidade genética.

1 Herds with heterogeneity of all parameters.

2 Herds with similar genetic means.

${ }^{3}$ Herds with phenotypic heterogeneity.

${ }^{4}$ Herds without heterogeneity.

5 Genetic connectness. 
dois grupos de classificação, principalmente quando a intensidade de seleção foi alta (5\% dos melhores touros).

Quando os rebanhos possuíam 100\% de conexidade genética, independentemente do tipo de heterogeneidade presente nos dados, as porcentagens de touros comuns nos dois grupos de classificação foram altas, variando de 73 a 100\% (Tabela 1). Portanto, a avaliação genética de touros foi pouco influenciada pela presença de heterogeneidade entre os rebanhos quando os dados apresentavam alta conexidade genética entre os níveis de variabilidade. Resultados semelhantes foram observados para a estrutura RSH. No entanto, para a estrutura de dados RHF, a presença de conexidade não aumentou a porcentagem de touros comuns entre os grupos, provavelmente em razão do efeito da heterogeneidade para variância residual, uma vez que, na estrutura de dados RHF, foi este tipo de variância que determinou a heterogeneidade para variâncias fenotípicas.

Weigel et al. (2001) estimaram parâmetros genéticos e valores genéticos de reprodutores utilizados em vários países para a característica produção de leite e encontraram grandes alterações na ordem de classificação dos touros geneticamente superiores, com base nos valores genéticos preditos para cada país. Segundo os autores, estes resultados podem refletir as diferenças nos sistemas de produção destes países, ou simplesmente uma carência de informação atribuída à pequena ligação genética entre os países ocasionada pelo uso de poucos touros em comum.

A posição real dos 15 melhores touros (seleção de 20\% dos touros) classificados com base nos valores genéticos preditos na análise de característica única para as estruturas de heterogeneidade RHTP e RSH é apresentada na Tabela 2. Os resultados para as estruturas de heterogeneidade RMGS e RHF foram similares aos apresentados para RSH.

Considerando os valores genéticos preditos na estrutura heterogeneidade RHTP e CG- 0 , muitos touros com menor potencial genético foram classificados entre os animais geneticamente superiores. Dez touros cuja posição real foi bem inferior à 15a colocação foram classificados, incorretamente, entre os 15 melhores touros (Tabela 2).

No entanto, quando os dados apresentaram $100 \%$ de conexidade genética (CG-100) ou não havia heterogeneidade entre rebanhos (RSH), os erros de classificação dos touros foram menores. Nestes casos, mesmo que continuem ocorrendo pequenas alterações na ordem de classificação dos touros, apenas alguns animais, com posição real inferior à 15a colocação, foram classificados entre os 15 melhores (Tabela 2).

Para verificar o efeito da heterogeneidade sobre a seleção de vacas, adotou-se o mesmo procedimento realizado
Tabela 2 - Posição real dos 15 melhores touros classificados com base nos valores genéticos preditos nas diferentes estruturas de heterogeneidade

Table 2 - Actual position of the 15 top bulls ranked by predicted breeding values in different structures of heterogeneity

\begin{tabular}{|c|c|c|c|c|}
\hline \multirow{3}{*}{$\begin{array}{l}\text { Ordem } \\
\text { Rank }\end{array}$} & \multicolumn{2}{|c|}{$\begin{array}{c}\mathrm{RHTP}^{1} \\
\text { НHАP }\end{array}$} & \multicolumn{2}{|c|}{$\begin{array}{l}\mathrm{RSH}^{2} \\
H W H\end{array}$} \\
\hline & $\mathrm{CG}-0^{3}$ & CG-100 & CG-0 & CG-100 \\
\hline & & & & \\
\hline $1 \underline{\mathrm{a}}$ & $1 \underline{\mathrm{a}}$ & 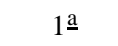 & $2 \underline{\mathrm{a}}$ & 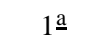 \\
\hline $2 \underline{a}$ & $28 \stackrel{\mathrm{a}}{ }$ & $2 \underline{a}$ & $3 \underline{a}$ & $2 \underline{a}$ \\
\hline $3 \underline{a}$ & $69 \underline{a}$ & $3 \underline{a}$ & $8 \underline{a}$ & $3 \underline{a}$ \\
\hline $4 \underline{a}$ & $44 \underline{\mathrm{a}}$ & $4 \underline{\mathrm{a}}$ & $4 \underline{\mathrm{a}}$ & $4 \underline{\mathrm{a}}$ \\
\hline $5 \underline{a}$ & $13 \underline{\mathrm{a}}$ & $6 \underline{a}$ & $1 \underline{\mathrm{a}}$ & $6 \underline{\mathrm{a}}$ \\
\hline $6 \underline{a}$ & $16 \underline{\mathrm{a}}$ & $8 \underline{a}$ & $5 \underline{a}$ & $5 \underline{\mathrm{a}}$ \\
\hline $7 \underline{\mathrm{a}}$ & $7 \underline{\mathrm{a}}$ & $5 \underline{\mathrm{a}}$ & $6 \underline{a}$ & $7 \underline{\mathrm{a}}$ \\
\hline $8^{\mathrm{a}}$ & $46^{\mathrm{a}}$ & $10 \underline{\mathrm{a}}$ & $11 \underline{\mathrm{a}}$ & $16 \underline{a}$ \\
\hline 9aㅡ & $50 \underline{\mathrm{a}}$ & $7 \underline{\mathrm{a}}$ & $14 \underline{\mathrm{a}}$ & $10 \underline{\mathrm{a}}$ \\
\hline $10 \underline{\mathrm{a}}$ & $6 \underline{\mathrm{a}}$ & $20 \underline{\mathrm{a}}$ & $13 \underline{\mathrm{a}}$ & $13 \underline{\mathrm{a}}$ \\
\hline $11 \underline{\mathrm{a}}$ & $17 \stackrel{\mathrm{a}}{ }$ & $14 \underline{\mathrm{a}}$ & $28 \stackrel{\mathrm{a}}{ }$ & $15 \underline{\mathrm{a}}$ \\
\hline $12 \underline{\mathrm{a}}$ & $5 \underline{a}$ & $11 \underline{\mathrm{a}}$ & $20 \underline{\mathrm{a}}$ & $12 \underline{\mathrm{a}}$ \\
\hline $13 \underline{\mathrm{a}}$ & 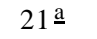 & $18 \underline{\mathrm{a}}$ & $21 \mathrm{a}$ & $11 \underline{\mathrm{a}}$ \\
\hline $14 \underline{\mathrm{a}}$ & $51 \underline{\mathrm{a}}$ & $33 \underline{\mathrm{a}}$ & $17 \underline{\mathrm{a}}$ & $20 \underline{\mathrm{a}}$ \\
\hline $15 \stackrel{\mathrm{a}}{ }$ & $23 \underline{\mathrm{a}}$ & $13 \underline{\mathrm{a}}$ & $15 \underline{a}$ & $8^{\mathrm{a}}$ \\
\hline
\end{tabular}

${ }^{1}$ Rebanhos com heterogeneidade em todos os parâmetros.

2 Rebanhos sem heterogeneidade.

${ }^{3}$ Conexidade genética.

1 Herds with heterogeneity of all parameters.

${ }^{2}$ Herds without heterogeneity.

${ }^{3}$ Genetic connectness.

para a classificação dos touros. Calculou-se a porcentagem de vacas comuns entre os dois grupos de animais classificados com base nos valores genéticos preditos na análise de característica única e com base nos valores genéticos verdadeiros para 5, 10 e 20\% das melhores vacas (Tabela 3 ).

Considerando a estrutura de heterogeneidade RHTP e CG-0, as porcentagens de vacas comuns entre os grupos de classificação foram muito baixas (variando de 14 a 26\%) e, com o aumento da conexidade genética entre os rebanhos, tiveram pequeno aumento, mas ainda continuaram baixas, não ultrapassando $32 \%$ de animais comuns entre os grupos de classificação para as $20 \%$ das melhores vacas (Tabela 3 ). Portanto, mesmo para dados com $100 \%$ de conexidade genética entre os níveis de variabilidade, a predição dos valores genéticos para vacas foi muito afetada pela presença da heterogeneidade entre os rebanhos para parâmetros genéticos e fenotípicos. Nestas condições, pequena porcentagem das vacas geneticamente superiores seria identificada corretamente pela avaliação genética.

Os resultados para as estruturas de heterogeneidade RMGS, RHF e RSH foram semelhantes. As porcentagens de vacas comuns entre os grupos de classificação variaram de 45 a $67 \%$. Para estas estruturas de dados, maior porcentagem das melhores vacas será corretamente identificada pela avaliação genética em relação aos dados com 
Tabela 3 - Porcentagens de animais em comum entre os grupos de classificação, com base no valor genético verdadeiro e no valor genético predito, para 5,10 e $20 \%$ das melhores vacas

Table 3 - Percentage of cows in common in the two groups ranked by true or predicted breeding values for 5,10 and $20 \%$ of selection

\begin{tabular}{lcccc}
\hline & \multicolumn{5}{c}{$\begin{array}{c}\text { Estrutura dos dados } \\
\text { Data structure }\end{array}$} \\
\cline { 2 - 5 } & RHTP $^{1}$ & RMGS $^{2}$ & RHF $^{3}$ & RSH $^{4}$ \\
& HHAP & $H S G M$ & $H P H$ & $H W H$ \\
\hline Seleção (\%) & \multicolumn{5}{c}{ CG-0 } \\
Selection(\%) & \multicolumn{5}{c}{ GC-0 } \\
\hline 5 & 14 & 51 & 52 & 55 \\
10 & 19 & 59 & 59 & 63 \\
20 & 26 & 64 & 67 & 65 \\
\hline Seleção (\%) & \multicolumn{5}{c}{ CG-100 } \\
Selection(\%) & \multicolumn{5}{c}{ GC-100 } \\
\hline 5 & 16 & 45 & 52 & \\
10 & 22 & 56 & 61 & 61 \\
20 & 32 & 64 & 67 & 67 \\
\hline
\end{tabular}

${ }_{1}^{1}$ Rebanhos com heterogeneidade em todos os parâmetros.

2 Rebanhos com médias genéticas similares.

${ }^{3}$ Rebanhos com heterogeneidade fenotípica.

${ }^{4}$ Rebanhos sem heterogeneidade.

5 Conexidade genética.

${ }^{1}$ Herds with heterogeneity of all parameters.

2 Herds with similar genetic means.

${ }^{3}$ Herds with phenotypic heterogeneity.

${ }^{4}$ Herds without heterogeneity.

${ }^{5}$ Genetic connectness.

heterogeneidade para todos os parâmetros. Também neste caso, o aumento na conexidade genética entre os níveis de variabilidade não aumentou a porcentagem de animais comuns entre os grupos de classificação. A ausência do efeito da conexidade genética sobre a avaliação de vacas era esperada, visto que as informações de determinada vaca e de suas progênies foram obtidas sempre dentro de um mesmo rebanho, independentemente do grau de conexidade dos dados.

Para todas as estruturas de dados, quanto mais intensa a seleção, menor a porcentagem de animais comuns entre os grupos de classificação, pois o efeito dos erros na ordem de classificação é mais evidente quando pequeno número de animais é selecionado.

O erro na classificação das vacas pode prejudicar a seleção e o progresso genético dentro dos rebanhos, mas o maior efeito da heterogeneidade sobre o progresso genético ocorre, principalmente, na seleção de vacas que serão mães de touros ou de vacas doadoras de embriões ou óvulos, uma vez que, neste caso, são altas as intensidades de seleção e utilização destas vacas, supostamente de elevado potencial genético. Assim, pequenas alterações na ordem de classificação das vacas poderiam determinar a eliminação de animais de alto valor genético e a seleção de animais com menor potencial genético.

A posição real de classificação das 15 melhores vacas classificadas com base nos valores genéticos preditos na análise de característica única para as estruturas de heterogeneidade RHTP e RSH é apresentada na Tabela 4. Os resultados para as estruturas de heterogeneidade RMGS e RHF foram similares aos apresentados para RSH.

Considerando a predição dos valores genéticos na presença de heterogeneidade genética e fenotípica (RHTP), pode-se afirmar que, entre as 15 melhores vacas, estão muitos animais de baixo valor genético cuja classificação real, considerando a classificação com base no valor genético verdadeiro, é muito inferior à identificada pela avaliação genética. Muitos animais cuja posição real ficou abaixo da $100^{a}$ colocação foram classificados entre as 15 melhores vacas (Tabela 4). Quando não houve heterogeneidade entre os rebanhos (RSH), embora os erros de classificação não tenham desaparecido, a magnitude destes erros reduziu bastante. Apenas alguns animais com posição real abaixo da $100^{a}$ colocação foram classificados entre as 15 melhores vacas (Tabela 4).

Esses resultados revelaram que a heterogeneidade entre rebanhos para médias genéticas afetou a acurácia da predição dos valores genéticos das vacas. Entretanto, a heterogeneidade para outros parâmetros, como variância genética, média fenotípica e variância fenotípica, não influenciou a predição dos valores genéticos das vacas.

O procedimento aplicado para verificar os efeitos da heterogeneidade sobre a seleção de touros e vacas também foi adotado para touros jovens. Também, neste caso, para a estrutura de heterogeneidade RHTP, foram encontradas as menores porcentagens de animais comuns entre os dois grupos de classificação (Tabela 5).

Para as estruturas sem heterogeneidade para média genética (RMGS, RHF e RSH), as porcentagens de animais comuns entre os grupos de classificação foram maiores, variando de 35 a $53 \%$ e de 47 a $62 \%$ para a seleção de 1 e $5 \%$ dos melhores touros jovens, respectivamente (Tabela 5).

Semelhantemente ao que ocorreu na seleção de vacas, para touros jovens, a porcentagem de animais comuns entre grupos de classificação foi menor quando a intensidade de seleção foi mais alta.

Para a maioria das estruturas de heterogeneidade, o aumento na conexidade genética entre os níveis de variabilidade não melhorou as predições dos valores genéticos dos touros jovens. As porcentagens de animais comuns entre os grupos de classificação foram similares para as estruturas de dados com 0 e $100 \%$ de conexidade genética, exceto para a estrutura de heterogeneidade RHTP e seleção 
Tabela 4 - Posição real de classificação das 15 melhores vacas classificadas com base nos valores genéticos preditos nas diferentes estruturas de heterogeneidade

Table 4 - Actual position of the 15 top cows ranked by predicted breeding values in different structures of heterogeneity

\begin{tabular}{|c|c|c|c|c|}
\hline \multirow[b]{2}{*}{$\begin{array}{l}\text { Ordem } \\
\text { Rank }\end{array}$} & \multicolumn{2}{|c|}{$\begin{array}{c}\mathrm{RHTP}^{1} \\
\text { НHАP }\end{array}$} & \multicolumn{2}{|c|}{$\begin{array}{l}\mathrm{RSH}^{2} \\
H W H\end{array}$} \\
\hline & $\begin{array}{c}\mathrm{CG}-0^{3} \\
G C-0\end{array}$ & $\begin{array}{c}\text { CG-100 } \\
G C-100\end{array}$ & $\begin{array}{l}\text { CG-0 } \\
G C-0\end{array}$ & $\begin{array}{c}\text { CG-100 } \\
G C-100\end{array}$ \\
\hline $1 \underline{\mathrm{a}}$ & $663 \underline{\underline{a}}$ & $4 \stackrel{\mathrm{a}}{-}$ & $3 \underline{a}$ & $4 \stackrel{\mathrm{a}}{ }$ \\
\hline $2 \underline{a}$ & $26 \underline{a}$ & $892 \underline{a}$ & $6 \underline{\mathrm{a}}$ & 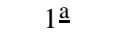 \\
\hline $3 \underline{a}$ & $357 \underline{a}$ & 5므 & $1 \underline{\mathrm{a}}$ & $2 \underline{a}$ \\
\hline $4 \underline{\mathrm{a}}$ & $15 \underline{\mathrm{a}}$ & $893^{\mathrm{a}}$ & $2 \mathrm{a}$ & $28 \underline{\text { a }}$ \\
\hline $5 \underline{\mathrm{a}}$ & $916^{\underline{a}}$ & $786^{\mathrm{a}}$ & $27 \underline{\text { a }}$ & $27 \underline{\text { a }}$ \\
\hline $6 \underline{\mathrm{a}}$ & $10 \underline{\mathrm{a}}$ & $310 \mathrm{a}$ & $12 \underline{\mathrm{a}}$ & $236 \mathrm{a}$ \\
\hline $7 \underline{a}$ & $324^{\mathrm{a}}$ & $518^{\mathrm{a}}$ & $139 \underline{a}$ & $10 \underline{\mathrm{a}}$ \\
\hline $8 \underline{a}$ & $296^{\underline{a}}$ & $23 \underline{a}$ & $5 \underline{a}$ & $129 \underline{a}$ \\
\hline 9啇 & $609 \underline{\underline{a}}$ & $312^{\underline{a}}$ & $88 \underline{\text { a }}$ & $128^{\mathrm{a}}$ \\
\hline $10 \underline{a}$ & $2 \underline{a}$ & $431 \underline{a}$ & $20 \underline{a}$ & $34 \underline{\mathrm{a}}$ \\
\hline $11 \underline{\mathrm{a}}$ & $559 \underline{a}$ & $262^{\mathrm{a}}$ & $59 \underline{\text { a }}$ & $17 \underline{\text { a }}$ \\
\hline $12 \stackrel{\mathrm{a}}{-}$ & $1857^{\mathrm{a}}$ & $2181^{\mathrm{a}}$ & $29 \underline{a}$ & $23 \underline{a}$ \\
\hline $13 \underline{\mathrm{a}}$ & $401 \stackrel{\mathrm{a}}{ }$ & 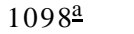 & $125 \underline{a}$ & 56 a \\
\hline $14 \underline{\mathrm{a}}$ & $333 \underline{\underline{a}}$ & 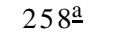 & $32 \underline{a}$ & $271 \underline{\mathrm{a}}$ \\
\hline $15 \underline{\mathrm{a}}$ & $11 \underline{\mathrm{a}}$ & $253 \underline{a}$ & $39 \underline{\text { a }}$ & $398 \underline{a}$ \\
\hline
\end{tabular}

${ }^{1}$ Rebanhos com heterogeneidade em todos os parâmetros.

${ }^{2}$ Rebanhos sem heterogeneidade.

3 Conexidade genética.

1 Herds with heterogeneity of all parameters.

2 Herds without heterogeneity.

${ }^{3}$ Genetic connectness.

Tabela 5 - Porcentagens de animais em comum entre os grupos de classificação com base no valor genético verdadeiro e no valor genético predito para 1 e $5 \%$ dos melhores touros jovens

Table 5 - Percentage of young bulls in common in the two groups ranked by true or predicted breeding values, for 1 and $5 \%$ of selection

\begin{tabular}{|c|c|c|c|c|}
\hline & \multicolumn{4}{|c|}{$\begin{array}{c}\text { Estrutura dos dados } \\
\text { Data structure }\end{array}$} \\
\hline & $\begin{array}{c}\mathrm{RHTP}^{1} \\
\text { HНAP }\end{array}$ & $\begin{array}{c}\mathrm{RMGS}^{2} \\
H S G M\end{array}$ & $\begin{array}{c}\mathrm{RHF}^{3} \\
H P H\end{array}$ & $\begin{array}{l}\mathrm{RSH}^{4} \\
\mathrm{HWH}\end{array}$ \\
\hline Seleção (\%) & \multicolumn{4}{|c|}{ CG- $-0^{5}$} \\
\hline Selection (\%) & \multicolumn{4}{|c|}{$G C-0$} \\
\hline 1 & 20 & 35 & 53 & 40 \\
\hline 5 & 24 & 47 & 58 & 58 \\
\hline Seleção (\%) & \multicolumn{4}{|c|}{ CG-100 } \\
\hline Selection (\%) & \multicolumn{4}{|c|}{$G C-100$} \\
\hline 1 & 20 & 53 & 50 & 43 \\
\hline 5 & 38 & 48 & 54 & 62 \\
\hline
\end{tabular}

${ }^{1}$ Rebanhos com heterogeneidade em todos os parâmetros.

2 Rebanhos com médias genéticas similares.

${ }^{3}$ Rebanhos com heterogeneidade fenotípica.

4 Rebanhos sem heterogeneidade.

5 Conexidade genética.

${ }^{1}$ Herds with heterogeneity of all parameters.

2 Herds with similar genetic means.

${ }^{3}$ Herds with phenotypic heterogeneity.

${ }^{4}$ Herds without heterogeneity.

${ }^{5}$ Genetic connectness.

de $5 \%$ dos touros jovens, na qual a porcentagem de animais comuns aumentou de 24 para $38 \%$, e, também, para a estrutura de heterogeneidade RMGS e seleção de $1 \%$ dos touros jovens, em que a porcentagem de animais comuns passou de 35 para 53\% quando houve conexidade genética entre os níveis de variabilidade.

O erro na classificação dos touros jovens pode comprometer o sucesso dos programas de melhoramento, pois é alto o investimento de tempo e de recursos para avaliações subseqüentes destes animais para as características reprodutivas, o teste de desempenho em centrais e o teste de progênies. Outro fator importante é a eliminação precoce de animais de alto valor genético e a seleção de animais com baixo potencial genético.

A posição real de classificação dos 15 melhores touros jovens classificados com base nos valores genéticos preditos na análise de característica única geral para as estruturas de heterogeneidade RHTP e RSH é apresentada na Tabela 6. Os resultados relativos às alterações na ordem de classificação dos animais para as estruturas de heterogeneidade RMGS e RHF foram similares aos apresentados para RSH.

$\mathrm{Na}$ análise dos dados na presença de heterogeneidade genética e fenotípica (RHTP), tanto para CG-0 como para CG-100, ocorreram grandes alterações na ordem de classificação dos animais. Muitos animais de baixo potencial genético foram classificados, incorretamente, entre os 15 melhores touros jovens. Por exemplo, considerando a classificação com base no valor genético verdadeiro, muitos animais cuja posição real é bem abaixo da 100 a colocação foram classificados, incorretamente, entre os 15 melhores touros jovens (Tabela 6).

$\mathrm{Na}$ ausência de heterogeneidade entre os rebanhos, os erros de classificação continuaram ocorrendo, porém, a magnitude das alterações na ordem de classificação foi bem menor. Apenas um animal com posição abaixo da $100^{a}$ colocação foi classificado incorretamente entre os 15 melhores touros jovens (Tabela 6).

Estes resultados sugerem que a heterogeneidade entre rebanhos para médias genéticas teve grande efeito sobre a acurácia da predição dos valores genéticos dos touros jo vens. Porém, quando a heterogeneidade entre os rebanhos ocorreu para outros parâmetros como variância genética, média fenotípica e variância fenotípica, os resultados das avaliações genéticas dos touros jovens foram próximos aos obtidos para dados sem heterogeneidade entre rebanhos.

Martins (2002), em trabalho de revisão bibliográfica, destacou como causas da heterogeneidade entre rebanhos as diferenças na composição genética da população causadas por seleção, pela composição racial, por diferenças na intensidade de seleção ou pela interação genótipo $\times$ ambiente. Vários outros autores relataram que a composição genética dos rebanhos também é um dos fatores responsáveis pela heterogeneidade entre rebanhos. Variâncias heterogêneas para grupos genéticos têm sido 
Tabela 6 - Posição real de classificação dos 15 melhores touros jovens classificados com base nos valores genéticos preditos nas diferentes estruturas de heterogeneidade

Table 6 - Actual position of the 15 top young bulls ranked by predicted breeding values in different structures of heterogeneity

\begin{tabular}{|c|c|c|c|c|}
\hline \multirow[b]{2}{*}{$\begin{array}{l}\text { Ordem } \\
\text { Rank }\end{array}$} & \multicolumn{2}{|c|}{$\begin{array}{c}\mathrm{RHTP}^{1} \\
\text { НHАP }\end{array}$} & \multicolumn{2}{|c|}{$\begin{array}{l}\mathrm{RSH}^{2} \\
H W H\end{array}$} \\
\hline & $\begin{array}{c}\mathrm{CG}-0^{3} \\
G C-0\end{array}$ & $\begin{array}{c}\text { CG-100 } \\
G C-100\end{array}$ & $\begin{array}{c}\text { CG-0 } \\
G C-0\end{array}$ & $\begin{array}{c}\text { CG-100 } \\
G C-100\end{array}$ \\
\hline $1 \underline{\mathrm{a}}$ & $1 \underline{\mathrm{a}}$ & $1 \underline{\mathrm{a}}$ & $1 \underline{\mathrm{a}}$ & $1 \underline{\mathrm{a}}$ \\
\hline $2 \underline{\mathrm{a}}$ & $188^{\mathrm{a}}$ & 9a & $36 \underline{a}$ & 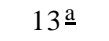 \\
\hline $3 \underline{a}$ & $3 \underline{a}$ & $598 \underline{a}$ & 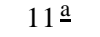 & 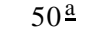 \\
\hline $4 \stackrel{\mathrm{a}}{-}$ & $110^{\underline{a}}$ & $18 \stackrel{\mathrm{a}}{ }$ & $29 \stackrel{a}{-}$ & $2 \underline{a}$ \\
\hline $5 \underline{a}$ & $8 \underline{\mathrm{a}}$ & $261 \underline{\mathrm{a}}$ & $4 \underline{\mathrm{a}}$ & $9 \underline{a}$ \\
\hline $6 \underline{a}$ & $873 \underline{a}$ & $34 \underline{a}$ & $27 \stackrel{a}{-}$ & $3 \underline{a}$ \\
\hline $7 \underline{\mathrm{a}}$ & $594 \underline{a}$ & $194 \underline{\mathrm{a}}$ & $42 \underline{\mathrm{a}}$ & $17 \underline{\mathrm{a}}$ \\
\hline $8^{\underline{a}}$ & $220^{\mathrm{a}}$ & $61 \stackrel{\mathrm{a}}{\underline{-1}}$ & $31 \stackrel{\mathrm{a}}{-}$ & $52 \underline{\mathrm{a}}$ \\
\hline $9 \underline{a}$ & $173^{\mathrm{a}}$ & $13 \underline{a}$ & 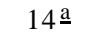 & $22 \underline{a}$ \\
\hline $10 \underline{a}$ & $1040 \underline{a}$ & $292 \underline{a}$ & $193 \underline{a}$ & $5 \underline{a}$ \\
\hline $11 \underline{a}$ & $10 \mathrm{a}$ & $42 \underline{a}$ & $69 \underline{a}$ & $60 \underline{a}$ \\
\hline $12 \underline{\mathrm{a}}$ & $754 \underline{\mathrm{a}}$ & $156^{\mathrm{a}}$ & $44 \stackrel{\mathrm{a}}{-}$ & $29 \stackrel{\mathrm{a}}{ }$ \\
\hline 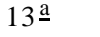 & $138 \underline{\mathrm{a}}$ & $473 \underline{a}$ & $72 \underline{a}$ & $34 \stackrel{a}{-}$ \\
\hline $14 \underline{\mathrm{a}}$ & $676^{\mathrm{a}}$ & $130^{\mathrm{a}}$ & $34 \underline{a}$ & $6 \underline{\mathrm{a}}$ \\
\hline $15 \underline{a}$ & $718 \underline{a}$ & $43 \underline{a}$ & $10 \underline{\mathrm{a}}$ & $10 \underline{a}$ \\
\hline
\end{tabular}

${ }^{1}$ Rebanhos com heterogeneidade em todos os parâmetros.

2 Rebanhos sem heterogeneidade.

3 Conexidade genética.

1 Herds with heterogeneity of all parameters.

2 Herds without heterogeneity.

${ }^{3}$ Genetic connectness.

encontradas em gado de corte para várias características (Rodriguez-Almeida et al., 1995; Nuñez-Domingues et al., 1995; Crews \& Franke, 1998; Oliveira et al., 2001). Estes mesmos autores também têm demonstrado a alteração na ordem de classificação dos animais quando considerada ou não a heterogeneidade de variância entre grupos genéticos.

Entre grupos genéticos, a média genética dos animais provavelmente não é homogênea. Assim, os resultados citados por esses autores e os descritos neste trabalho evidenciam que o problema da heterogeneidade entre rebanhos sobre as avaliações genéticas é ocasionado, basicamente, pela presença de médias genéticas diferentes entre rebanhos.

\section{Conclusões}

A heterogeneidade entre rebanhos para parâmetros como variância genética, média fenotípica e variância fenotípica não prejudica a avaliação genética dos animais.

A presença de médias genéticas diferentes entre rebanhos prejudica a acurácia da predição dos valores genéticos de touros e, principalmente, de vacas e touros jovens, acarretando grandes erros na classificação dos animais geneticamente superiores.
Na presença de heterogeneidade para média genética, a conexidade genética entre rebanhos melhorou a predição dos valores genéticos de touros, porém, os erros na classificação de vacas e touros jovens ainda permaneceram altos.

\section{Agradecimento}

Ao Departamento de Zootecnia e ao Programa de PósGraduação em Genética e Melhoramento da Universidade Federal de Viçosa (UFV), pela infra-estrutura disponibilizada para a execução deste trabalho, e à Coordenação de Aperfeiçoamento do Pessoal de Nível Superior (CAPES), pela concessão da bolsa de pesquisa.

\section{Literatura Citada}

BOLDMAN, K.G.; FREEMAN, A.E. Adjustment for heterogeneity of variances by herd production level in dairy cow and sire evaluation. Journal of Dairy Science, v.73, n.2, p.503-512, 1990.

CANAVESI, F.; SCHAEFFER, L.R.; BURNSIDE, E.B. et al. Sire $\times$ herd interaction effect when variance across herds are heterogeneous. I. Expected genetic progress. Journal of Animal Breeding Genetic, v.112, p. 95-106, 1995.

CREWS, D.H.; FRANKE, D.E. Heterogeneity of variances for carcass traits by percentage Brahman inheritance. Journal of Animal Science, v.76, p.1803-1809, 1998.

LITTEL, R.C.; FREUND, R.J.; SPECTOR, P.C. SAS system for linear models. 3.ed. Cary: SAS Institute Inc., 1993. 329p.

MARTINS, E.N. Avaliação genética e heterogeneidade de variância. In: REUNIÃO ANUAL DA SOCIEDADE BRASILEIRA DE ZOOTECNIA, 39., 2002, Recife. Anais... Sociedade Brasileira de Zootecnia, 2002. (CD-ROM)

MEUWISSEN, T.H.E.; Van Der WERF, J.H.J. impact of heterogeneous within herd variances on dairy cattle breeding. Livestock Production Science, v.33, p.31-41, 1993.

NUÑEZ-DOMINGUEZ, R.; Van VLECK, L.D.; CUNDIFF, L.V. et al. Prediction of genetic values of sires for growth traits of crossbred cattle using a multivariate animal models with heterogeneous variances. Journal of Animal Science, v.73, p.2940-2950, 1995.

OLIVEIRA, C.A.L.; MARTINS, E.N.; FREITAS, A.R. et al. Heterogeneidade de variâncias nos grupos genéticos formadores da raça Canchim. Revista Brasileira de Zootecnia, v.30, n.4, p.1212-1219, 2001.

RODRIGUEZ-ALMEIDA, F.A.; Van VLECK, L.D.; CUNDIFF, L.V Heterogeneity of variance by sire breed, sex and dam breed in 200 and 365-day weights of beef cattle from top cross experiment. Journal of Animal Science, v.73, n.9, p.25792588, 1995.

TORRES, R.A.; BERGMANN, J.A.G.; COSTA, C.N. et al. Heterogeneidade de variância e avaliação genética de bovinos da raça Holandesa no Brasil. Revista Brasileira de Zootecnia, v.29, n.4, p.1050-1059, 2000.

WEIGEL, K.A.; REKAYA, R. A multiple-trait herd cluster model for international dairy sires evaluation. Journal of Dairy Science, v.83, n.4, p.815-821, 2000

WEIGEL, K.A.; REKAYA, R.; ZWALD, N.R. et al. International genetic evaluation of dairy sires using a multiple-trait model with individual animal performance records. Journal of Dairy Science, v.84, n.12, p.2789-2795, 2001

Recebido: $14 / 03 / 05$ Aprovado: 19/05/06 